\title{
Clinical experience with a novel subcutaneous implantable defibrillator system in a single center
}

\author{
Lara Dabiri Abkenari - Dominic A. M. J. Theuns • Suzanne D. A. Valk • \\ Yves Van Belle · Natasja M. de Groot • David Haitsma • Agnes Muskens-Heemskerk • \\ Tamas Szili-Torok • Luc Jordaens
}

Received: 2 November 2010/ Accepted: 23 February 2011/Published online: 17 March 2011

(c) The Author(s) 2011. This article is published with open access at Springerlink.com

\begin{abstract}
Background Implantable cardioverter-defibrillators (ICDs) reduce mortality in both primary and secondary prevention, but are associated with substantial short- and long-term morbidity. A totally subcutaneous ICD (S-ICD) system has been developed. We report the initial clinical experience of the first 31 patients implanted at our hospital.

Methods All patients had an ICD indication according to the ACC/AHA/ESC guidelines. The first 11 patients were part of the reported CE trial. The implantation was performed without fluoroscopy. The device was implanted subcutaneously in the anterior axillary line, with a parasternal lead tunneled from the xiphoid to the manubrial-sternal junction. Ventricular fibrillation (VF) was induced to assess detection accuracy and defibrillation efficacy using $65 \mathrm{~J}$ shocks.

Results Post-implant, 52 sustained episodes of VF were induced. Sensitivity was $100 \%$ and induced conversion efficacy was $100 \%$ (with standard polarity in 29 patients). Mean time to therapy was $13.9 \pm 2.5 \mathrm{~s}$ (range 11-21.6 s). Late procedure-related complications were observed in 2 of the first 11 implantations (lead migration). During followup, spontaneous ventricular arrhythmias occurred in four patients, with accurate detection of all episodes. Inappropriate therapy was observed in five patients. Recurrences were prevented with reprogramming.
\end{abstract}

L. Dabiri Abkenari · D. A. M. J. Theuns ·

S. D. A. Valk · Y. Van Belle - N. M. de Groot - D. Haitsma .

A. Muskens-Heemskerk · T. Szili-Torok

Department of Cardiology, Erasmus MC,

Rotterdam, The Netherlands

L. Jordaens $(\bowtie)$

Department of Electrophysiology, Ba 581, Erasmus MC,

PO Box 2040, 3000 CA Rotterdam, The Netherlands

e-mail: 1.jordaens@erasmusmc.nl
Conclusions The S-ICD system can be implanted without the use of fluoroscopy by using anatomical landmarks only. Episodes of VF were accurately detected using subcutaneous signals, and all induced and clinical episodes were successfully converted. The S-ICD system is a viable alternative to conventional ICD systems for selected patients.

Keywords Defibrillator - Sudden cardiac death . Ventricular fibrillation - Ventricular tachycardia . Arrhythmias

\section{Introduction}

Implantable cardioverter-defibrillators (ICDs) have become standard therapy to prevent sudden cardiac death, but have recently been associated with serious short- and long-term morbidity, such as lead failure, premature depletion, endocarditis and inappropriate shocks. A totally subcutaneous ICD (S-ICD) system has been developed to address some of these concerns [1]. We report the initial clinical experience of the first 31 patients who received this device in our department.

\section{Methods}

Patient population

Files of all patients receiving a totally subcutaneous cardioverter-defibrillator in our institution were reviewed for this report. Patients were selected for an S-ICD if they had an ICD indication according to the ACC/AHA/ESC 2006 guidelines for primary or secondary prevention. A total of 
31 patients ( 24 males, 7 females) received the S-ICD system. The patient's mean age was $53 \pm 4$ years. Left ventricular ejection fraction was $38 \pm 15 \%$, which was relatively high due to the number of patients with prophylactic implantations for channelopathy or genetic disease (32\% had LVEF $>50 \%$ ). Coronary artery disease was present in 18 patients and the indication was primary prophylaxis in 21 patients. Coronary artery disease was present in 18 patients (58\%), dilated cardiomyopathy in 4 (13\%), Brugada disease in 2, idiopathic ventricular fibrillation in 3, idiopathic VT in 2 and non-compaction cardiomyopathy and valvular disease were diagnosed each in 1 patient. Demographic data are given in Tables 1, 2.

ECG showed an infarction in 13 patients. The mean PR interval was $165.2 \pm 31.6 \mathrm{~ms}$ with one patient in atrial fibrillation at the time of implant. The mean QRS width was $105 \pm 16.2 \mathrm{~ms}$. No QT abnormalities were observed; three patients had an incomplete left bundle branch block (LBBB), three had a complete LBBB and one an intermittent RBBB.

In addition, we checked a $24 \mathrm{~h}$ recording to exclude evident bradycardia or high degree block at the time of implant. The mean heart rate during the $24 \mathrm{~h}$ Holter was $70 \pm 8$ beats $/ \mathrm{min}$. The minimal and maximal heart rates were $48 \pm 5$ and $111 \pm 17$ beats $/ \mathrm{min}$, respectively; the mean longest $\mathrm{RR}$ interval was $1,281 \pm 149 \mathrm{~ms}$. Two patients previously had a transvenous ICD explantation, one for hematoma and painful deep venous thrombosis, and the other for a pace-sense lead break.

Most patients were admitted on the day of the procedure and discharged on the following day. All gave informed

Table 1 Clinical characteristics of patients implanted with an S-ICD

\begin{tabular}{ll}
\hline Characteristics & Value \\
\hline Number & 31 \\
Age (years \pm SD) & $53 \pm 16$ \\
Gender (male/female) & $24 / 7$ \\
Height $(\mathrm{cm} \pm \mathrm{SD})$ & $175 \pm 10$ \\
Weight $(\mathrm{kg} \pm \mathrm{SD})$ & $79 \pm 17$ \\
BMI $\left(\mathrm{kg} / \mathrm{m}^{2} \pm \mathrm{SD}\right)$ & $29 \pm 16$ \\
LVEF $(\% \pm \mathrm{SD})$ & $38.8 \pm 15$ \\
CAD $(n)$ & $18(58 \%)$ \\
Primary prevention $(n)$ & $21(67 \%)$ \\
ECG-PR $>200 \mathrm{~ms}(n)$ & $4(13 \%)$ \\
QRS $>120 \mathrm{~ms}(n)$ & $4(13 \%)$ \\
Holter & \\
Mean HR (beats/min $\pm \mathrm{SD})$ & $70 \pm 8$ \\
Minimal HR (beats/min $\pm \mathrm{SD})$ & $48 \pm 5$ \\
Longest RR interval $(\mathrm{ms} \pm \mathrm{SD})$ & $1,281 \pm 149$ \\
\hline
\end{tabular}

$B M I$ body mass index, $C A D$ coronary disease, $H R$ heart rate, $L V E F$ left ventricular ejection fraction, $S D$ standard deviation, $S-I C D$ subcutaneous defibrillator, $n$ number
Table 2 Patient characteristics

\begin{tabular}{lcll}
\hline Pathology & $\begin{array}{l}\text { Patient } \\
\text { number }\end{array}$ & $\begin{array}{l}\text { Percentage } \\
\text { Average } \\
\text { LVEF } \\
(\%)^{\mathrm{a}}\end{array}$ \\
\hline $\begin{array}{l}\text { Coronary artery disease } \\
\begin{array}{l}\text { Idiopathic VF (genetic } \\
\text { determination) }\end{array}\end{array}$ & 3 & 9.5 & 60.6 \\
$\begin{array}{l}\text { Idiopathic VT } \\
\text { Brugada syndrome }\end{array}$ & 2 & 6.5 & 65 \\
$\begin{array}{l}\text { Idiopathic dilated } \\
\text { cardiomyopathy }\end{array}$ & 2 & 6.5 & 62 \\
$\quad$ Valvular disease & 4 & 13 & 25.5 \\
$\quad$ Non-compaction & 1 & 3.25 & 54 \\
$\quad$ cardiomyopathy & 1 & 3.25 & 21 \\
\hline
\end{tabular}

$V T$ ventricular tachycardia, $V F$ ventricular fibrillation

${ }^{a}$ LVEF determined by echocardiography, nuclear scan or magnetic resonance imaging

consent and were aware of the innovative aspects, limitations and potential advantages and disadvantages of the device.

Beta-blocking agents and antiarrhythmic drugs were continued. Prior to implantation, oral anticoagulation was discontinued until the INR was normalized. Patients with frequent ventricular arrhythmias necessitating ICD therapy and those with a pacing indication were not considered for this device. The first 11 patients were included in the study by Bardy et al. [1] which only reported a follow-up of 3 months.

The device and its programming

The subcutaneous ICD system (model SQ-RX 1010; Cameron Health Inc., San Clemente, CA, USA), comprises a pulse generator and a subcutaneous lead (model Q-Trak 3010; Cameron Health Inc.) with two sensing electrodes to record the electrical activity of the heart. These electrodes represent three vector projections of electrical conduction occurring through the heart (A-to-CAN, B-to-CAN, and A-to-B) (Fig. 1). The S-ICD system operates by analyzing both the rate and morphological characteristics of the detected rhythm. The device can be programmed as single zone or dual zone. The device calculates heart rate based on the average of the last four intervals. As soon as the heart rate crosses the lowest programmed detection zone, further analysis is performed to determine whether therapy is required. Therapy consists of an $80 \mathrm{~J}$ shock, with potential temporary transthoracic back-up pacing for $30 \mathrm{~s}$.

Implantation procedure

General anesthesia was used for the first two patients, and for the rest local anesthesia was given in combination with 
Fig. 1 S-ICD vector configuration shown on the $\mathrm{X}$-ray of a patient after implantation. The drawing on the right shows how the QRS and the $T$-wave are assessed by the device and the physician before implantation, to ensure that a correct vector will be selected

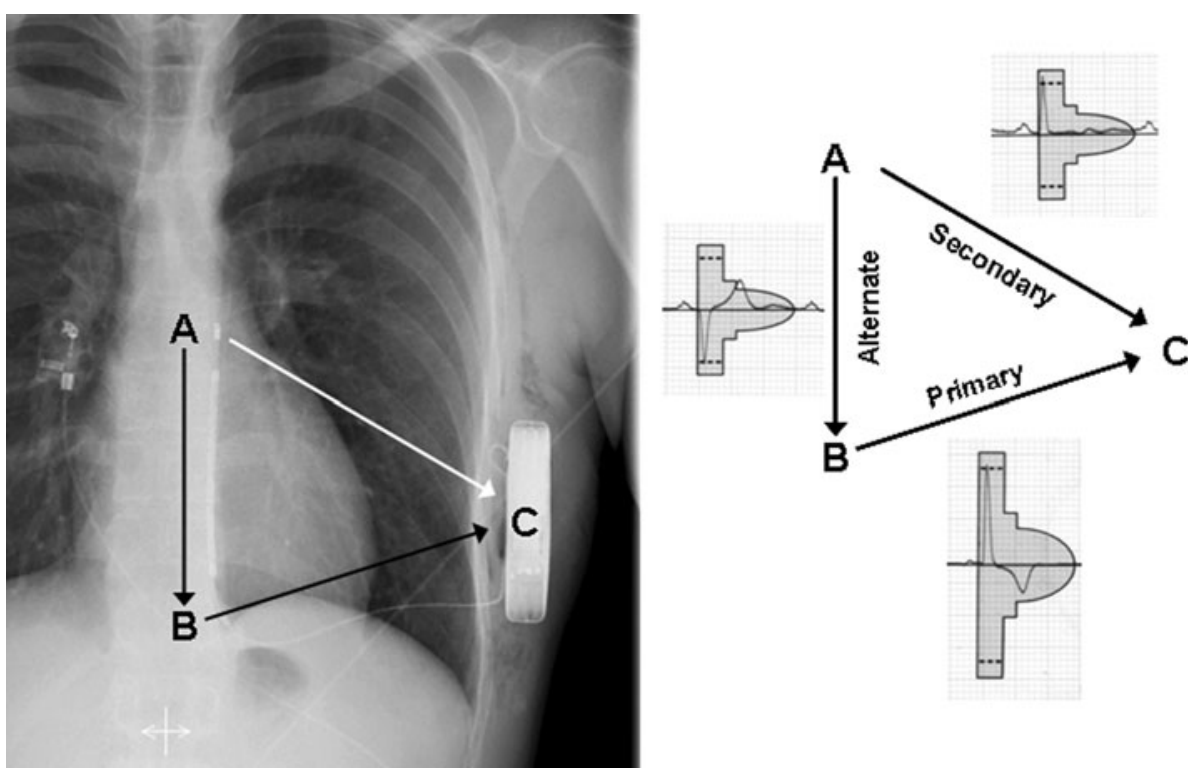

sedation, as done routinely for transvenous implantations. Antibiotic prophylaxis was administered $1 \mathrm{~h}$ before the procedure. The subcutaneous defibrillator was implanted without fluoroscopy using anatomical landmarks only. The pocket was created in the left axillary region at the level of the sixth rib (Fig. 2). The parasternal defibrillation lead was placed left of the sternal midline using small incisions at the xiphoid and at the sternal-manubrium junction. The lead was pulled into position by tunneling from xiphoid to the pocket, and subsequently from the xiphoid to the sternal-manubrium junction. Fixation of the lead was performed by a suture at the tip in all patients. After the first 15 patients, an additional suture sleeve was used at the xiphoid position. For the defibrillation efficacy testing ("DFT"), etomidate was given before VF was induced. Two consecutive shocks of $65 \mathrm{~J}$ were required as per protocol in the initial 13 patients. Polarity was reversed in case of failure. After the initial series, only one effective shock was performed. One day after the procedure, a chest $\mathrm{X}$-ray was taken to control ICD position.

Follow-up

After discharge, patients were seen in the device outpatient clinic of our institute. After implantation, patients were seen pre-discharge, 10 days and 2 months after implantation. Subsequently, patients underwent a regular ICD interrogation at 6-monthly intervals or shorter if clinically required. At each follow-up visit, arrhythmic events with stored subcutaneous electrocardiograms (S-ECGs) were retrieved from the device's memory.

\section{Results}

Patients

A total number of 31 patients received an S-ICD between December 2008, and October 2010.

Implantation

The implantations were usually done within $100 \mathrm{~min}$ (average of $101 \pm 33 \mathrm{~min}$ ) including the DFT (with $3 \mathrm{~min}$ between each attempt and up to three attempts before external defibrillation was used). They were performed by a group of three electrophysiologists, including one in the first year of training, without using fluoroscopy. No particular surgical problems were encountered. The procedures under local anesthesia were relatively well tolerated; only one patient was put under deep sedation for excessive pain. The most time-consuming step was the making of a lateral pocket. The DFT was performed after positioning of the generator in the pocket without closing it completely. All patients could be converted from induced VF to sinus rhythm, with the initial polarity (Fig. 3a), except for two patients in whom reversed polarity was programmed. Non-sustained AF was induced by the shock in three cases, but reverted spontaneously before the end of the procedure and never triggered further activation of the device. Post-shock bradycardia occurred in one patient, which was resolved with pacing by the device (Fig. 3b). All devices were programmed with maximal shocks $(80 \mathrm{~J})$. 


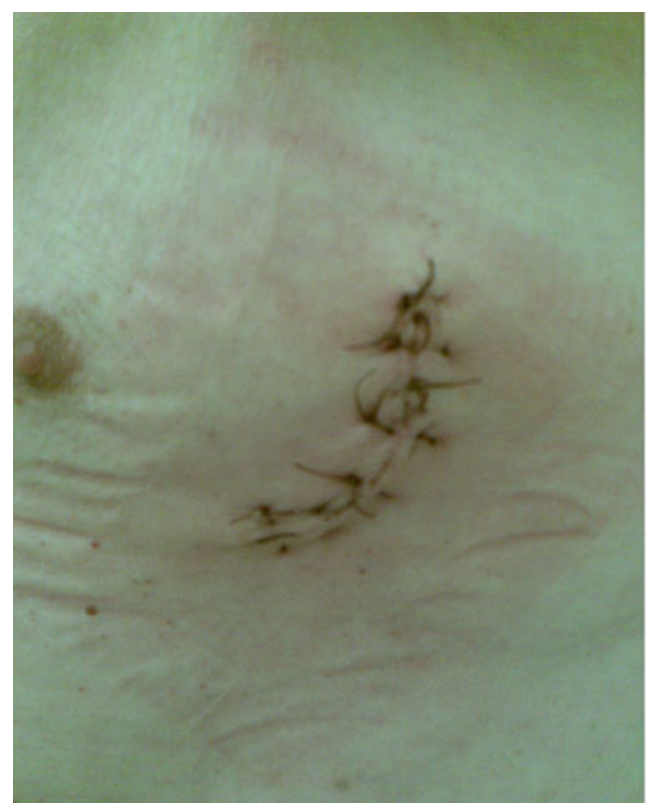

Fig. 2 Lateral (at the left) and frontal (at the right) view of a patient who received an S-ICD many years after coronary artery bypass grafting (with a midsternal scar). The device and the lead are almost

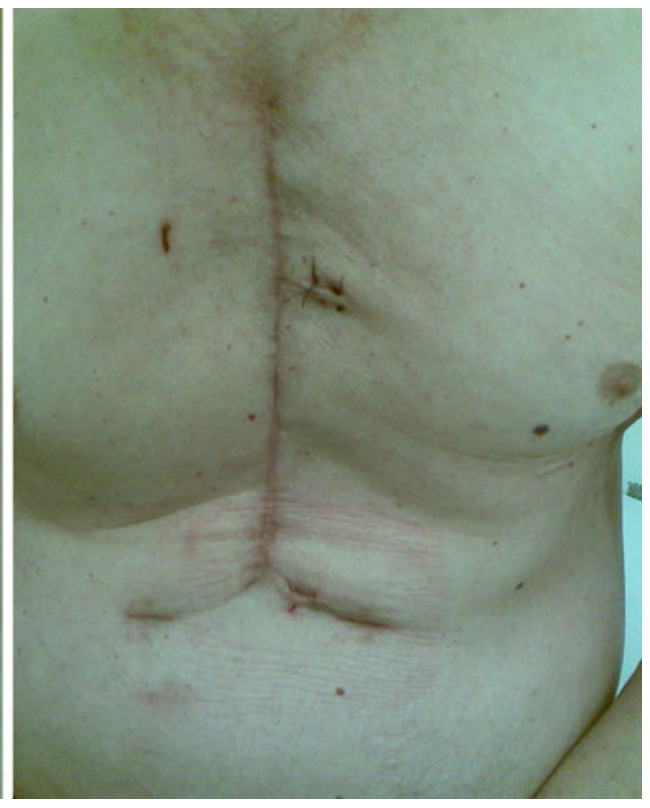

invisible. The lateral incision was closed with seven discontinuous stitches. The manubrial and xyphoid wounds are barely visible

A

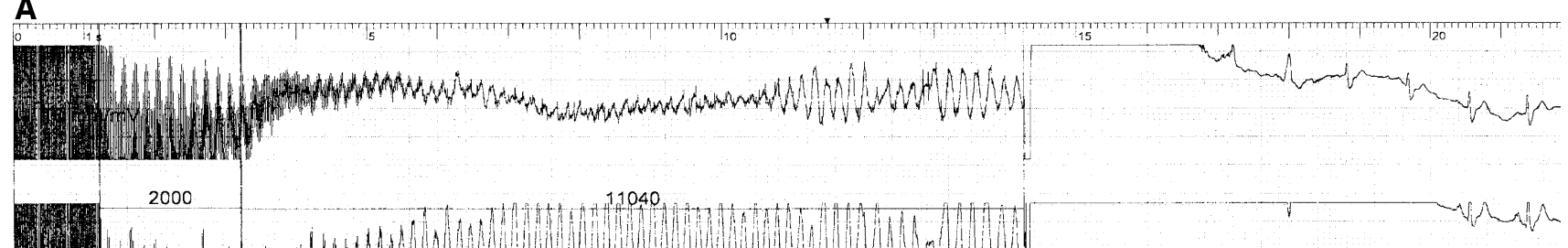
2.

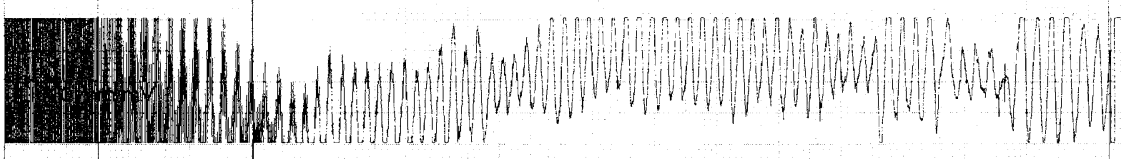

B

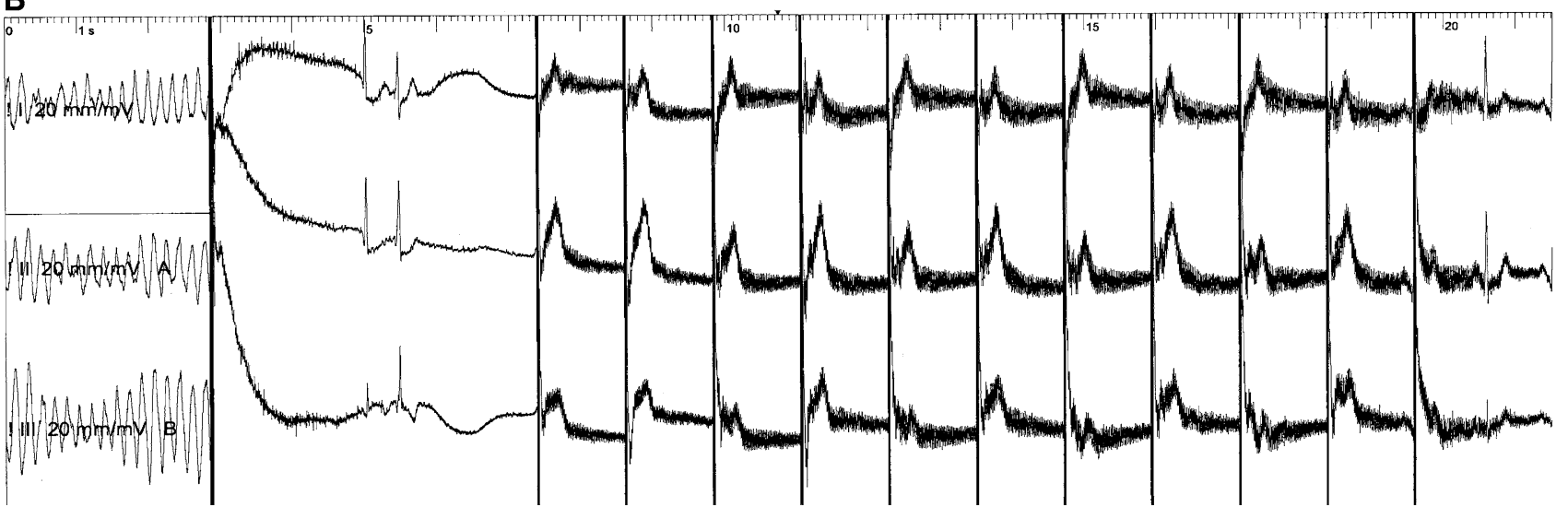

Fig. 3 a Detection of induced VF and subsequent shock during defibrillation threshold testing. b Transthoracic post-shock pacing after conversion of induced ventricular fibrillation 
Follow-up

No record of unexplained syncope or death in our group exists till now, with a median follow-up of 286 days (range 30-638) and a total number of 350 patient-months under observation (till 1 October 2010).

\section{Surgical problems}

A late procedure-related complication was observed in two of the first 15 patients implanted, with dislocation of the lead due to migration of the electrode. One of these patients suffered from two inappropriate shocks due to myopotentials. The other was detected with an X-ray during routine control. Initial implanting recommendations did not utilize an electrode suture sleeve at the xyphoid level, but this was integrated into the implantation protocol thereafter. In both cases, repositioning of the wires was done under local anesthesia, with the addition of a dedicated suture sleeve (Fig. 4) No further inappropriate therapy was noted in the patient $(239+$ days since implant). There was one case of pocket infection with tissue necrosis, which led to explantation of the entire system (without difficulty). This occurred in a 14-year-old boy in a cachectic state after treatment with extracorporeal membrane oxygenation for severe heart failure and an ongoing skin infection. The implantation was carried out despite the infectious risk to allow revalidation and because his life vest had become intolerable to him.

\section{Inappropriate shocks}

Five patients had inappropriate shocks. Two were clearly due to myopotentials, one during vigorous sawing and the above-mentioned lead displacement. These events occurred prior to the software update, which specifically addressed sensing during myopotentials. Since update, no inappropriate therapy has occurred $(506+$ days). There was a case of oversensing during heavy coughing and one case of double counting after the appearance of a complete right bundle branch block. The fifth patient had an inappropriate shock on a very fast sinus tachycardia, the sense vectors were evaluated and a new vector was selected with an updated template. No further inappropriate therapy has been noted in this patient. These events are summarized in Table 3.

\section{Arrhythmias}

All ventricular arrhythmias, detected in four patients, were treated successfully (Fig. 5). This included 11 appropriate
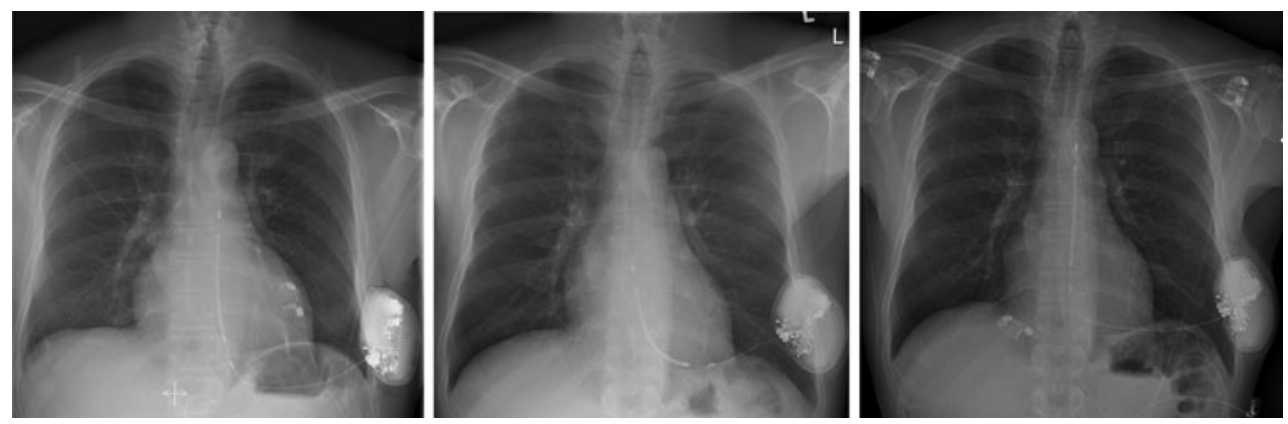

Fig. 4 Initial position of lead (at the left), obvious lead displacement (in the middle) and after correction (at the right)

Table 3 Inappropriate shocks

\begin{tabular}{|c|c|c|c|c|c|c|}
\hline $\begin{array}{l}\text { Inappropriate } \\
\text { shocks }(n)\end{array}$ & $\begin{array}{l}\text { Patient } \\
\text { number }\end{array}$ & Cause & Solution & $\begin{array}{l}\text { Recurrence } \\
\text { Y/N }\end{array}$ & $\begin{array}{l}\text { Interval from } \\
\text { implant } \\
\text { (days) }\end{array}$ & $\begin{array}{l}\text { "Arrhythmia" } \\
\text { cycle length } \\
\text { detected (ms) }\end{array}$ \\
\hline 1 & 1 & Myopotential detection (noise) & Software upgrade & $\mathrm{N}$ & 237 & 160 \\
\hline 2 & 1 & $\begin{array}{l}\text { Noise(myopotentials) from lead } \\
\text { dislodgment }\end{array}$ & Lead reposition & $\mathrm{N}$ & 461 & 260 \\
\hline 15 & 1 & T-wave oversensing (new RBBB) & New template for EGM made & $\mathrm{N}$ & 59 & 200 \\
\hline 1 & 1 & Double counting & Alternate vector selection & $\mathrm{N}$ & 421 & 300 \\
\hline 1 & 1 & T-wave oversensing & Alternate vector selection & $\mathrm{N}$ & 625 & 300 \\
\hline
\end{tabular}

$R B B B$ right bundle branch block, $E G M$ electrogram, $Y$ yes, $N$ no 
Fig. 5 Detection and termination of spontaneous fast ventricular tachycardia (coded as T) with a shock, $18 \mathrm{~s}$ after initiation of arrhythmia

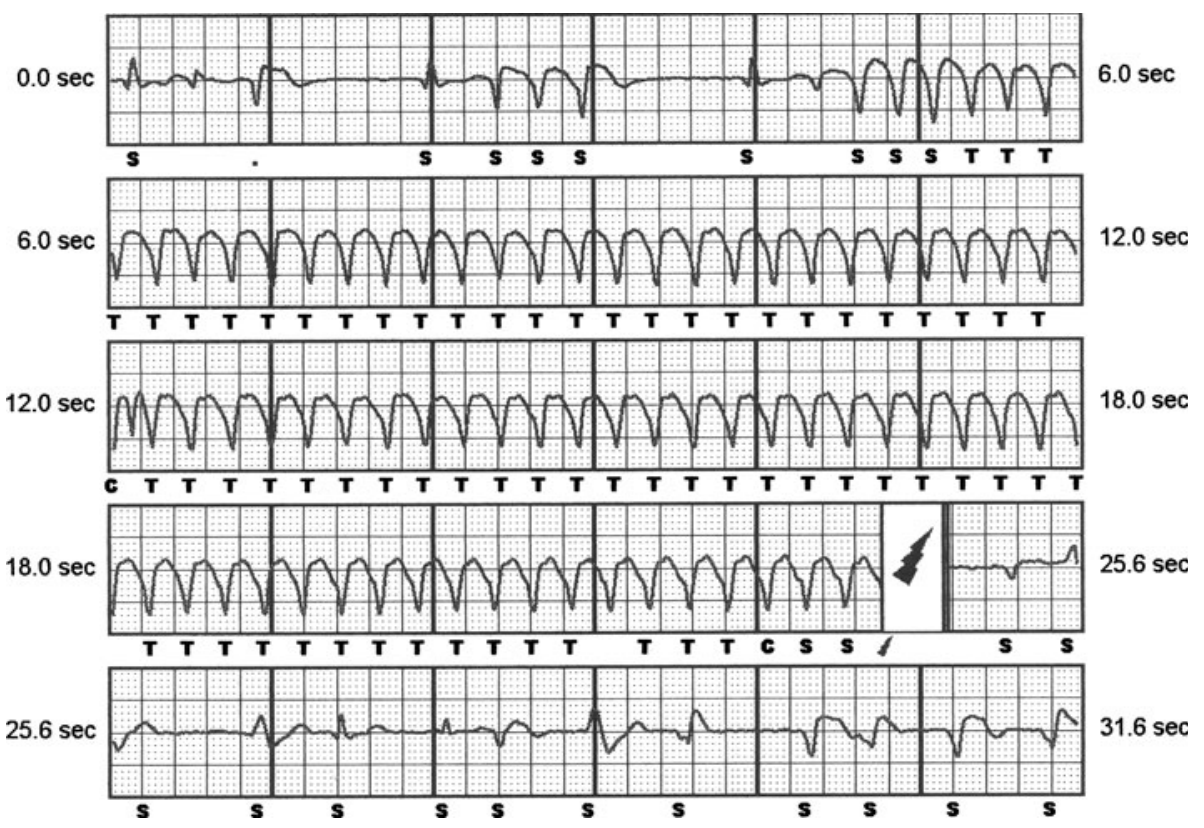

shocks for ventricular fibrillation in the patient with the infection and correct detection of four episodes of nonsustained arrhythmias in the VF zone. In one patient, six shocks were delivered for ventricular tachycardia (VT) [cycle lengths (CL) of 240, 250 and $260 \mathrm{~ms}$ ], with correct detection of seven episodes of non-sustained VT (CL 220, 240 and $260 \mathrm{~ms}$ ). In this patient, the S-ICD was explanted and replaced by a transvenous system to allow ATP and also pacing for a later acquired symptomatic bradycardia. There was detection of a non-sustained VT in two other patients.

\section{Discussion}

Implantable cardiac defibrillators are indicated in patients with a high risk for sudden death. This is confirmed after the initial scientific studies by extensive meta-analysis and reconfirmed in most international and national guidelines [2-5]. Nevertheless, it became recently clear that some disadvantages of ICDs can outweigh the advantages of a prophylactic implantation [6]. Therefore it seems reasonable to consider less invasive systems to offer the advantage of internal defibrillation to patients with a moderate risk.

General advantages of SQ systems

The procedural advantages of subcutaneous systems are evident [8]. The risk of vascular damage during implantation is minimized. This implies that implantation is possible in patients with congenital heart disease or after extensive thrombosis or infection of the venous access. The subcutaneous system was effective in all cases in our experience. This was true for induced and spontaneous arrhythmias. In our patient group, for two patients this ICD was selected due to a complication from an endovascular ICD (lead break and deep vein thrombosis). After a complication implicating a transvenous device, patients are reluctant to undergo a second implantation, and the stress of possible complications related to explantation makes the subcutaneous system even more attractive. Indeed, there is no risk of endocarditis with infection and cardiac or venous damage with an explantation. After implantation, patients do not have to limit arm movements for 6 weeks, but only for about a week to 10 days until the stitches are removed. After implantation, there are no particular limitations in movement of the arms, and this is indeed appreciated by younger and more active patients. In our experience, this has been of particular interest to younger patients with a primary prevention indication in hereditary diseases such as Brugada syndrome or genetic idiopathic ventricular fibrillation. A recent study involving 61 patients diagnosed with Brugada syndrome suggests that programming a single high-rate VF zone may be associated with reduced inappropriate defibrillator discharges [9].

Transvenous implantation can otherwise be associated with right-sided venous thrombosis, endocarditis, surgical complications, pocket bleeding and pneumothorax when subclavian puncture is necessary [10]. Further, it became clear in the recent years that lead related problems were very often present and it is even said that after 10 years only $80 \%$ of leads still function as anticipated [7,11]. We did not observe any of these disadvantages in our patients. However, it must be noted that the longevity of subcutaneous leads is not yet well established. The only surgical 
problems we encountered were two lead migrations. This occurred in the era before a suture sleeve was applied at the xyphoid, which prevented migration in all the later implanted patients. Further, we had one hematoma in a very thin and generally ill patient in whom a life vest was given because a transvenous device was judged to be an impossible approach.

Specific advantages in comparison with conventional devices

The subcutaneous device has incorporated some new technology specifically for interpretation of the electrocardiogram and claims good arrhythmia discrimination in the absence of transvenous leads. We observed inappropriate shocks in the beginning of this series. All events were easily explained and by upgrading the template or the software, spurious shocks could be further prevented. Whether this incidence is higher or lower, in comparison with conventional devices, has to be analyzed over a longer period or in a randomized study. At this moment, we have the impression that the incidents are reasonably further avoided with optimal programming, better vector selection and the introduction of the extra suture sleeve [12].

Specific disadvantages of subcutaneous systems

A potential disadvantage is that no bradycardia pacing and anti-tachycardia pacing is possible. The absence of pacing could more or less be considered an advantage for this therapy, as it was shown that a certain amount of pacing is associated with a decrease in left ventricular function. This was initially shown for pacemaker patients and also for patients requiring ICD therapy $[13,14]$. No pacing also implies no anti-tachycardia pacing. This could potentially be of lesser concern when prophylactic implantations are considered in particular where the target is indeed ventricular fibrillation. We only had one (secondary prophylaxis) patient in whom it was regretted that no ATP facilities were present. He had also developed symptomatic bradycardia.

The implantation procedure can in itself be quite painful, there are three scars instead of one, and the box is heavier and larger and in a more sensitive part of the thorax. Patients reported pain and the need for pain medication, but it was mostly limited to the first week after implantation.

\section{Patient selection}

We did not change our selection process greatly for determination of suitable candidates for this device. An important concern is the need for pacing or ATP. However, by using Holter recordings and analyzing them in a very conventional way, we are able to exclude patients with evident or symptomatic bradycardia, high degree AV block or having pauses so that pacing function was necessary in addition to the ICD. Further, patients with monomorphic tachycardia who could have been candidates for ATP were not selected. Another consideration could be that the presence of transvenous leads might be a disadvantage when an ablation may be indicated. Recently, it was proposed that a pacemaker may be associated with the S-ICD if necessary [15].

\section{Limitations}

This single center experience was not randomized and shows the short term, initial experience of our group. This implies that no real data on long-term performance of the device and its longevity were available.

\section{Conclusions}

We had the opportunity to use a new device, which was associated with mostly reversible complications and an efficacy that seemed reasonable for the reported follow-up. Inappropriate shocks occurred during this short observation time, all cases were elucidated and an efficient solution (so far) was found. It is worthwhile to perform larger trials to gain experience with this new prophylactic device. There was only one death in our group due to an aggressive lung carcinoma, and there were no episodes of unexplained syncope or hospitalizations for heart failure. This device is also a potential alternative for patients who have had an ICD-related complication, have a limited vascular access or are reluctant to undergo a new implantation.

Open Access This article is distributed under the terms of the Creative Commons Attribution Noncommercial License which permits any noncommercial use, distribution, and reproduction in any medium, provided the original author(s) and source are credited.

\section{References}

1. Bardy GH, Smith WM, Hood MA, Crozier IG, Melton IC, Jordaens L et al (2010) An entirely subcutaneous only implantable cardioverter defibrillator. Ned Engl J Med 363:36-44

2. Moss AJ, Hall WJ, Cannom DS, Daubert JP, Higgins SL, Klein H, Levine JH, Saksena S, Waldo AL, Wilber D, Brown MW, Heo M (1996) Improved survival with an implanted defibrillator in patients with coronary disease at high risk for ventricular arrhythmia. Multicenter Automatic Defibrillator Implantation Trial Investigators. N Engl J Med 335:1933-1940

3. Moss AJ, Zareba W, Hall WJ, Klein H, Wilber DJ, Cannom DS, Daubert JP, Higgins SL, Brown MW, Andrews ML (2002) Multicenter Automatic Defibrillator Implantation Trial II 
Investigators. Prophylactic implantation of a defibrillator in patients with myocardial infarction and reduced ejection fraction. N Engl J Med 346:877-883

4. Bardy GH, Lee KL, Mark DB, Poole JE, Packer DL, Boineau R, Domanski M, Troutman C, Anderson J, Johnson G, McNulty SE, Clapp-Channing N, Davidson-Ray LD, Fraulo ES, Fishbein DP, Luceri RM, lp JH (2005) Sudden Cardiac Death in Heart Failure Trial (SCD-HeFT) Investigators. Amiodarone or an implantable cardioverter-defibrillator for congestive heart failure. N Engl J Med 352:225-237

5. Zipes DP, Camm AJ et al (2006) ACC/AHA/ESC 2006 guidelines for management of patients with ventricular arrhythmias and the prevention of sudden cardiac death. Europace 8:746-837

6. Epstein AE et al (2008) ACC/AHA/HRS 2008 guidelines for device-based therapy of cardiac rhythm abnormalities: executive summary. Heart Rhythm 5:934-955

7. Hauser RG, Hayes DL, Epstein AE, Cannom DS, Vlay SC, Song SL, Tyers GF (2006) Multicenter experience with failed and recalled implantable cardioverter-defibrillator pulse generators. Heart Rhythm 3:640-644

8. Remmelts HH, Meine M, Loh P, Hauer R, Doevendans PA, van Herwerden LA, Hopmans TE, Ellerbroek PM (2009) Infection after ICD implantation: operating room versus cardiac catheterisation laboratory. Neth Heart J 17:95-100

9. Veltmann C, Kuschyk J, Schimpf R, Streitner F, Schoene N, Borggrefe M, Wolpert C (2010) Prevention of inappropriate ICD shocks in patients with Brugada syndrome. Clin Res Cardiol 99:37-44

10. Kleemann T, Becker T, Doenges K, Vater M et al. (2007) Annual rate of transvenous defibrillation lead defects in implantable cardioverter-defibrillators over a period of $>10$ years. Circulation 115:2474-2480

11. Theuns DAMJ, Klootwijk APJ, Goedhart DM, Jordaens LJLM (2004) Prevention of inappropriate therapy in implantable cardioverter-defibrillators: results of a prospective, randomized study of tachyarrhythmia detection algorithms. J Am Coll Cardiol 44:2362-2367

12. Nielsen JC, Kristensen L, Andersen HR, Mortensen PT, Pedersen OL, Pedersen AK (2003) A randomized comparison of atrial and dual-chamber pacing in 177 consecutive patients with sick sinus syndrome: echocardiographic and clinical outcome. J Am Coll Cardiol 42:614-623

13. Wilkoff BL, Cook JR, Epstein AE, Greene HL, Hallstrom AP, Hsia H, Kutalek SP, Sharma A (2002) Dual chamber and VVI implantable defibrillator trial investigators. dual-chamber pacing or ventricular backup pacing in patients with an implantable defibrillator. JAMA 288:3115-3123

14. Grimm W, Plachta E, Maisch B (2006) Antitachycardia pacing for spontaneous rapid ventricular tachycardia in patients with prophylactic cardioverter-defibrillator therapy. Pacing Clin Electrophysiol 29:759-764

15. van Opstal J, Geskes G, Debie L. A (2010) completely subcutaneous implantable cardioverter defibrillator system functioning simultaneously with an endocardial implantable cardioverter defibrillator programmed as pacemaker. Europace 12 (Epub ahead of print) 